\title{
Optimal Relay-Subset Selection and Time-Allocation in Decode-and-Forward Cooperative Networks
}

\author{
Elzbieta Beres and Raviraj Adve \\ Dept. of Elec. and Comp. Eng. \\ University of Toronto \\ 10 King's College Road \\ Toronto, ON M5S 3G4, Canada \\ email: ela.beres@utoronto.ca,rsadve@comm.utoronto.ca
}

\begin{abstract}
We present the optimal relay-subset selection and transmission-time for a decode-and-forward, half-duplex cooperative network of arbitrary size. The resource allocation is obtained by maximizing over the rates obtained for each possible subset of active relays, and the unique time allocation for each set can be obtained by solving a linear system of equations. We also present a simple recursive algorithm for the optimization problem which reduces the computational load of finding the required matrix inverses, and reduces the number of required iterations. Our results, in terms of outage rate, confirm the benefit of adding potential relays to a small network and the diminishing marginal returns for a larger network. We also show that optimizing over the channel resources ensures that more relays are active over a larger SNR range, and that linear network constellations significantly outperform grid constellations. Through simulations, the optimization is shown to be robust to node numbering.
\end{abstract}

\section{INTRODUCTION}

Cooperation has become a popular technique to implement diversity in the absence of multiple antennas at receiving and transmitting nodes [1]-[3]. In this context, resource allocation in cooperative networks has recently become an active research area, and has been investigated under many scenarios and metrics. In this paper, we address the problem of resource allocation, in terms of channel resources (time or bandwidth), in multi-relay networks with arbitrary connections. We describe the contributions of the paper in detail after a brief review of the pertinent literature.

For the single-relay case, several works have dealt with various aspects of resource allocation, in terms of power and/or bandwidth and time. Yao et al. determine the optimal power and time allocation for relayed transmissions specifically in the low-power regime [4]. Larsson and Cao present various strategies for allocating power and channel resources under energy constraints [5]. For the channel resource allocation problem, however, the authors consider selection combining only and do not address the scenario of joint decoding of the source and relay signals. The works in [6]-[8] address the problem of power and channel resource allocation under sum average power constraints. Optimal time and bandwidth allocation using 
instantaneous and average channel conditions is obtained using power control in [9]. Channel resource allocation under fixed power is developed in [10]

In networks with multiple relays, the available literature can be classified into two groups: networks where relays do not communicate with one another (parallel-relay networks), and networks without restrictions on relay communication (arbitrarily-connected networks). Resource allocation for the former has been addressed in [11]-[14]. Ibrahimi and Liang develop the optimal power allocation for a multi-relay cooperative OFDMA amplify-and-forward (AF) system [11]. By maximizing the channel mutual information, Anghel et al. find the optimal power allocation for multiple parallel relays in AF networks [12], [13]. A more general solution is given in [14] where the authors give the optimal power and channel resource allocation for a parallel-relay network with individual power constraints on the nodes.

To the best of our knowledge, the problem of channel resource allocation for arbitrarily-connected networks and dedicated multiple access has not been addressed in the literature. In general, works in the area of multi-relay systems with arbitrary links generally neglect the bandwidth penalty arising from multiple hops by assuming either full-duplex nodes, a bandwidth-unconstrained system, or the availability of channel phase information at the transmitter [15]-[27].

These assumptions, however, are not realistic for practical wireless networks, where nodes are likely to be half-duplex, phase information is very difficult to obtain at the transmitter, and bandwidth is a scarce resource. To fill this void, in this paper we investigate the problem of resource allocation in a bandwidth-constrained, cooperative, decode-and-forward (DF), wireless network, and consider the most general setting where multiple relays can transmit can cooperate with each other in transmitting information between source and destination. In this setting, we address the joint problem of optimal selection of a relaying subset and allocation of time resources to the selected relays. The resource allocation is framed in the context of mesh networks of relatively simple and inexpensive nodes. We concentrate on resource allocation in terms of transmission time only, removing power allocation from the optimization; we further simply the problem by considering orthogonal transmissions. This is motivated by the need to reduce complexity, allowing for nodes which can implement the resource allocation simply by switching on and off. For a system without power allocation, a solution to this problem provides an upper bound on cooperative performance in multi-relay network where dedicated channels are assigned for each source transmission. 
To the best of our knowledge, no other work provides a solution to time-allocation for an arbitrarily connected cooperative network. The solution can be interpreted as a generalization of the opportunistic protocol presented by Gunduz and Erkip, where the relay is active only when it increases the outage rate [6]. In terms of the resource allocation solution, it is also a generalization of the solution in [10], where channel resource allocation is determined under fixed power for a three-node DF network. The solution can also be interpreted as a generalization of node selection [3], [28]-[30] under relaxed transmission constraints, where transmission can occur on multiple time-slots and relays can communicate with one another.

This paper is structured as follows. Section III describes the system model. In Section III and IV we develop the proposed resource allocation scheme and present a significantly simplified recursive implementation. Simulation results are presented in Section $\mathrm{V}$ and concluding remarks are presented in Section VI

\section{SyStem MODEL}

We consider a mesh network of static nodes comprising a source and destination node and $N$ potential relays. The inter-node channel powers are denoted as $\left|a_{i j}\right|^{2}$, where $i$ and $j$ represent the source node $s$, relay nodes $r_{k}, k=1 \ldots N$, or the destination node $d$. They are assumed independent of each other and are modelled as flat, slowly-fading and exponential with parameter $\lambda . \lambda$ is inversely proportional to the average channel power and is a function of inter-node distance, $d_{i j}$, through the path loss exponent $p_{a}$, e.g., $1 / \lambda_{s d}=\left(1 / d_{s d}^{p_{a}}\right)$, and $1 / \lambda_{r_{k} d}=\left(1 / d_{r_{k} d}^{p_{a}}\right)$. We do not include shadowing into the fading model, although this can easily be incorporated on an instantaneous basis. Because the nodes are static, the channels are assumed to change very slowly with time; we thus assume knowledge of all channel gains (although not channel phases) at a centralizing unit. This knowledge is essential to our resource allocation scheme.

With the aim of designing simple and cheap nodes, we assume half-duplex channels and orthogonal transmissions, which greatly simplifies receiver design. The relays are assumed to be numbered in some convenient order such that relay $r_{j}$ transmits after $r_{i}$ if $j>i$. For example, the relays may be in a linear constellation as shown in Figure 1, We also assume the DF cooperation strategy with independent codebooks, which allow for the optimization of system resources (see [31] for an overview on current coding methods for nodes using DF). Note that repetition coding does not allow for this resource allocation.

With these assumptions, the cooperation framework for the $N$-relay fully-connected network is as follows. The half-duplex constraint precludes the relays from transmitting and receiving simultaneously 
on the same channel, and the unavailability of forward-channel phase information at the nodes precludes the nodes from simultaneous transmissions. The transmission between the source and destination is thus divided into $N+1$ time-slots, of duration $t_{0}, t_{1}, \ldots, t_{N}$, with $t_{0}+t_{1}+\ldots+t_{N}=1$. In the first time-slot, the source transmits its information to all the nodes. The first relay, $r_{1}$, decodes this information and the remaining $N$ relays and the destination store the information for future processing. In the second slot, of duration $t_{1}$, the first relay re-transmits the information using an independent codebook, the second relay decodes the information from the first relay and the source, and the remaining $N-1$ relays and the destination store the information for further processing. In general, each relay $r_{k}$ decodes information from the source and from the previous relays $r_{1} \ldots r_{k-1}$ up to and including time-slot $t_{k-1}$. This process continues until all relays have transmitted and the destination attempts to decode the information.

Assuming that each node uses power $P$ and $W \mathrm{~Hz}$ per transmission (noting that although each node transmits for a different amount of time, the symbol durations and thus the corresponding bandwidth used by each node is the same), the signal to noise ratio (SNR) at node $j$ resulting from transmission from node $i$ can be written as $\mathrm{SNR}_{i j}=\frac{P}{N_{0} W}\left|a_{i j}\right|^{2}$, where $N_{0}$ is the noise spectral density. In the rest of the paper, we use the short-hand notation $L_{i j}$ to denote $\log _{2}\left(1+\mathrm{SNR}_{i j}\right)$, the capacity of the corresponding channel.

\section{Optimal Resource Allocation and Relay Selection}

In this section, we solve the joint problem of resource allocation and relay selection for the network discussed above. Essentially, we give the optimum values of $t_{i}, i=0 \ldots N$, such that the achievable rate between source and destination is maximized. We begin here with a fully-connected network, where each node is linked to all other nodes through a non-zero channel.

\section{A. Fully Connected Network}

Consider a source-destination pair communicating with the help of $N$ relays. Assuming that each relay is active, the mutual information at each relay and destination can be written as

$$
\begin{aligned}
I_{1}\left(t_{0}\right) & =t_{0} L_{s r_{1}} \\
I_{k}\left(t_{0}, t_{1}, t_{2}, \ldots, t_{k-1}\right) & =t_{0} L_{s r_{k}}+t_{1} L_{r_{1} r_{k}}+\ldots+t_{k-1} L_{r_{k-1} r_{k}}, \\
I_{D}\left(t_{0}, t_{1}, t_{2}, \ldots, t_{k}, \ldots, t_{N-1}, t_{N}\right) & =t_{0} L_{s d}+t_{1} L_{r_{1} d}+\ldots+t_{k-1} L_{r_{k-1} d}+\ldots+t_{N} L_{r_{N} d}
\end{aligned}
$$


where $I_{k}$ and $I_{D}$ denote the mutual information at relay $r_{k}$ and the destination, respectively.

With all $N$ relays cooperating, the maximum achievable rate under orthogonal transmissions is the minimum of the mutual information obtained at each individual relay node:

$$
\begin{aligned}
R_{N}=\max _{t_{0}, \ldots, t_{N}} \min & \left\{I_{1}\left(t_{0}\right), I_{2}\left(t_{0}, t_{1}\right), \ldots, I_{k-1}\left(t_{0}, \ldots, t_{k-2}\right), I_{k}\left(t_{0}, \ldots, t_{k-1}\right), I_{k+1}\left(t_{0}, \ldots, t_{k}\right),\right. \\
& \left.\ldots, I_{N}\left(t_{0}, \ldots, t_{k}, \ldots, t_{N-1}\right), I_{D}\left(t_{0}, \ldots, t_{k}, \ldots, t_{N-1}, t_{N}\right)\right\}, \\
\text { such that } \quad & t_{i} \geq 0, \quad \forall i, \\
& t_{0}+t_{1}+\ldots t_{N} \leq 1 .
\end{aligned}
$$

The above expression is a straightforward generalization of the cut-set bound for the single-relay network. This generalization maintains orthogonal transmissions for each relay, a model which represents practical networks with simple nodes that cannot implement complex interference cancelation. We use this model as the basis of the optimization in the rest of this paper. We note, however, that because each relay transmits using an orthogonal channel, $R_{N}$ is clearly not the channel capacity. For literature on the channel capacity of arbitrarily-connected networks, we direct the reader to [32]-[37] for full-duplex relays, and [38] for half-duplex relays.

For reasons that will soon become clear, let us consider the case with relay $r_{k}$ removed from the network. The maximum achievable rate $R_{N-1}^{k}$ becomes

$$
\begin{aligned}
R_{N-1}^{k}=\max _{t_{0}, \ldots, t_{k-1}, t_{k+1}, \ldots t_{N}} \min & \left\{I_{1}\left(t_{0}\right) \ldots, I_{k-1}\left(t_{0}, \ldots, t_{k-2}\right), I_{k+1}\left(t_{0}, \ldots, t_{k-1}\right),\right. \\
& \left.\ldots, I_{D}\left(t_{0}, \ldots, t_{k-1}, t_{k+1}, \ldots, t_{N}\right)\right\} \\
\text { such that } & t_{i} \geq 0, \quad \forall i \\
& t_{0}+\ldots t_{k-1}+t_{k+1}+\ldots t_{N} \leq 1 .
\end{aligned}
$$

Removing relay $r_{k}$ is thus equivalent to removing $t_{k}$ and $I_{k}$ from the optimization. [We use the subscript in $R_{N-1}^{k}$ to denote the maximum number of potentially active relays, and the superscript to denote the relay removed]. The maximum rate at which the source can transmit to the destination can thus be written as the maximum of the rate obtained by using all $N$ relays, and the rate obtained by successively removing each relay:

$$
R_{T}=\max \left\{R_{N}, R_{N-1}^{1}, R_{N-1}^{2}, \ldots, R_{N-1}^{N}\right\}
$$


If $R_{T}=R_{N-1}^{k}$, the maximum rate can be obtained by iterating through (4) and (5), successively removing a relay each step. Note that obtaining $R_{N-1}^{k}$ includes the cases where two or more relays are removed. In theory, therefore, all $2^{N}$ possible cases must be checked.

Let $\left(t_{0}^{*}, t_{1}^{*}, \ldots, t_{N}^{*}\right)$ denote the resource allocation that solves the optimization problem. We begin an outline of the solution to the optimization problem in (4), (5) and (6) with the following proposition.

Proposition 1: With a maximum number of potential relays $N$, the maximum achievable rate $R_{T}=R_{N}$ only if $t_{k}^{*} \neq 0, \forall k$. Otherwise, if $t_{k}^{*}=0, R_{T}=R_{N-1}^{k}$.

Proof: With exactly $N$ active relays, and with $k<n<N$, the resulting rate can be written explicitly as:

$$
\begin{aligned}
R_{N}=\max _{t_{0}, \ldots, t_{N}} \min & \left\{\left(t_{0} L_{s r_{1}}\right),\left(t_{0} L_{s r_{2}}+t_{1} L_{r_{1} r_{2}}\right), \ldots,\left(t_{0} L_{s r_{k}}+\ldots+t_{k-1} L_{r_{k-1} r_{k}}\right),\right. \\
& \left(t_{0} L_{s r_{n}}+\ldots+t_{k-1} L_{r_{k-1} r_{n}}+t_{k} L_{r_{k} r_{n}}+t_{k+1} L_{r_{k+1} r_{n}} \ldots t_{n-1} L_{r_{n-1} r_{n}}\right), \ldots \\
& \left.\left(t_{0} L_{s d}+\ldots+t_{k-1} L_{r_{k-1} r_{d}}+t_{k} L_{r_{k} r_{d}}+t_{k+1} L_{r_{k+1} r_{d}}+\ldots t_{N} L_{r_{N} r_{d}}\right)\right\}
\end{aligned}
$$

Setting $t_{k}=0$ gives

$$
\begin{aligned}
R_{N}=\max _{t_{0}, \ldots, t_{k-1}, t_{k+1}, \ldots t_{N}} \min & \left\{\left(t_{0} L_{s r_{1}}\right),\left(t_{0} L_{s r_{2}}+t_{1} L_{r_{1} r_{2}}\right), \ldots,\left(t_{0} L_{s r_{k}}+\ldots+t_{k-1} L_{r_{k-1} r_{k}}\right),\right. \\
& \left(t_{0} L_{s r_{n}}+\ldots+t_{k-1} L_{r_{k-1} r_{n}}+t_{k+1} L_{r_{k+1} r_{n}} \ldots t_{n-1} L_{r_{n-1} r_{n}}\right), \ldots, \\
& \left.\left(t_{0} L_{s d}+\ldots+t_{k-1} L_{r_{k-1} r_{d}}+t_{k+1} L_{r_{k+1} r_{d}}+\ldots t_{N} L_{r_{N} r_{d}}\right)\right\} \\
\leq \max _{t_{0}, \ldots, t_{k-1}, t_{k+1}, \ldots t_{N}} \min \{ & \left\{\left(t_{0} L_{s r_{1}}\right),\left(t_{0} L_{s r_{2}}+t_{1} L_{r_{1} r_{2}}\right), \ldots,\right. \\
& \left(t_{0} L_{s r_{n}}+\ldots+t_{k-1} L_{r_{k-1} r_{n}}+t_{k+1} L_{r_{k+1} r_{n}} \ldots t_{n-1} L_{r_{n-1} r_{n}}\right), \ldots, \\
& \left.\left(t_{0} L_{s d}+\ldots+t_{k-1} L_{r_{k-1} r_{d}}+t_{k+1} L_{r_{k+1} r_{d}}+\ldots t_{N} L_{r_{N} r_{d}}\right)\right\} \\
& =R_{N-1}^{k-1},
\end{aligned}
$$

since (9) has one fewer term in the minimization than (8).

To solve the optimization problem of (4) we thus require only the critical points for which $t_{k}^{*} \neq 0, \forall k$. In the following proposition, we show that for each $R_{N}$, i.e., given a set of potential relays, only one solution satisfies $t_{k}^{*} \neq 0, \forall k$.

Proposition 2: The unique solution to the optimization problem in the minimization in (4) for which $t_{k}^{*} \neq 0, \forall k$ is given by $I_{1}\left(t_{1}\right)=I_{2}\left(t_{1}, t_{2}\right)=\ldots=I_{N}\left(t_{1}, \ldots, t_{N}\right)=I_{D}\left(t_{1}, \ldots, t_{N}\right)$.

Proof: We consider all possible critical points obtained from the optimization in (4). The points are 
obtained either by maximizing each individual term in (4) or by intersecting all possible combinations of the terms in (4). We show that the only solution leading to non-zero solutions results from intersecting every term in (4).

The critical points for the optimization problem can be obtained by solving the following:

1) Maximize the individual terms in (4) except $I_{d}\left(t_{0}, \ldots, t_{N}\right)$ :

$$
\forall k \leq N, \quad \max _{t_{0}, \ldots, t_{k-1}} I_{k}\left(t_{0}, \ldots t_{k-1}\right) \quad \text { s.t. } \quad t_{0}+\ldots+t_{k-1} \leq 1
$$

Because the optimization is not over $t_{m}, \forall k \leq m \leq N$, the solution to this problem clearly has all $t_{m}=0, \forall k \leq m \leq N$, and thus cannot be a solution to the overall optimization problem.

2) Maximize $I_{d}\left(t_{0}, \ldots, t_{N}\right)$ :

$$
\max _{t_{0}, \ldots, t_{N}} I_{d}\left(t_{0}, \ldots t_{N}\right)=\max _{t_{0}, \ldots, t_{N}}\left\{t_{0} L_{s d}+\ldots+t_{N} L_{r_{N} r_{d}}\right\}, \quad \text { s.t. } \quad t_{0}+\ldots+t_{N} \leq 1
$$

In this case, all variables are included in the optimization. It is easy to show, however, that this function is maximized by selecting the largest $L$ value, i.e., evaluating the Kuhn-Tucker conditions leads to a solution of the form $t_{m}=1, t_{k}=0, \forall k \neq m$, where $m=\arg \max _{k}\left\{L_{s d}, L_{r_{1} d}, \ldots, L_{r_{k} d}, \ldots, L_{r_{N} d}\right\}$. Therefore, this solution is also not a solution to the overall optimization problem.

3) Maximize the function that results from the intersection of all possible combinations of the functions $I_{k}$. Let $\mathcal{M}$ denote all possible subsets of $\{1 \ldots N\} . \mathcal{M}$ then contains $2^{N}$ such subsets, i.e., $|\mathcal{M}|=2^{N}$. Consider one such subset $\delta_{k}=\left(m_{1}, m_{2}, \ldots, m_{k}\right)$, with $m_{1}<m_{2}<m_{k}$. One critical point then is

$$
\max _{t_{0}, \ldots, t_{m_{k-1}}} I_{m_{k}}\left(t_{0}, \ldots t_{m_{k-1}}\right)
$$

such that

$$
I_{m_{1}}\left(t_{0}, \ldots t_{m_{1}-1}\right)=I_{m_{2}}\left(t_{0}, \ldots t_{m_{2}-1}\right)=\ldots=I_{m_{k}}\left(t_{0}, \ldots t_{m_{k}-1}\right)
$$

This optimization then gets repeated for all sets $\delta_{k} \in \mathcal{M}$. In all but one combination, this optimization is not over all the variables $\left\{t_{0}, \ldots t_{N}\right\}$. As in point (1), this maximization also leads to $t_{k}=0$ for some value of $k$.

4) Maximize the intersection of all terms in (4):

$$
I_{1}\left(t_{0}\right)=I_{2}\left(t_{0}, t_{1}\right)=\ldots=I_{N}\left(t_{0}, \ldots, t_{N-1}\right)=I_{d}\left(t_{0}, \ldots, t_{N}\right)
$$


This is the only case that leads to $t_{k} \neq 0, \forall k=0 \ldots N$.

Essentially, this proposition shows that if all $N$ relays are to contribute, all terms in the minimization in (4) must be equal. This proposition applies to any value of $N$. Therefore, if the optimal solution has $k<N$ relays, an expression like (4) can be written for those $k$ relays.

\section{B. Optimal solution}

The linear system of equations in (15) has a simple solution. Setting each equation to a constant, solving for the vector of unknowns $\mathbf{t}=\left(t_{0} \ldots t_{N}\right)$ and normalizing, we obtain

$$
\mathbf{L}_{\mathbf{N}+\mathbf{1}} \mathbf{t}_{N+1}=\mathbf{1}_{N+1}, \Rightarrow \mathbf{t}_{N+1}=\frac{\mathbf{L}_{N+1}^{-1} \mathbf{1}_{N+1}}{\left\|\mathbf{L}_{N+1}^{-1} \mathbf{1}_{N+1}\right\|_{1}}=\frac{\mathbf{L}_{N+1}^{-1} \mathbf{1}_{N+1}}{\mathbf{1}_{N+1}^{T} \mathbf{L}_{N+1}^{-1} \mathbf{1}_{N+1}}
$$

where $\|\mathbf{v}\|_{1}$ denotes the sum of the elements of $\mathbf{v}$, i.e., the 1-norm. $\mathbf{1}_{N+1}$ is the length- $(N+1)$ vector of ones and $\mathbf{L}_{N+1}$ is the $(N+1) \times(N+1)$ rate matrix

$$
\mathbf{L}_{N+1}=\left[\begin{array}{ccccc}
L_{s r_{1}} & 0 & 0 & \ldots & 0 \\
L_{s r_{2}} & L_{r_{1} r_{2}} & 0 & \ldots & 0 \\
L_{s r_{3}} & L_{r_{1} r_{3}} & L_{r_{2} r_{3}} & \ldots & 0 \\
\vdots & \vdots & \vdots & \ddots & 0 \\
L_{s d} & L_{r_{1} d} & L_{r_{2} d} & \ldots & L_{r_{N} d}
\end{array}\right]
$$

The solution in (16) does not guarantee that the constraint $t_{k}>0 \quad \forall k=0 \ldots N$ is satisfied. To ensure that only solutions for which this constraint is satisfied are considered, we again consider the set $\mathcal{M}$. Each entry in the set corresponds to a rate matrix, $\mathbf{L}_{m}$, similar to that in (17), formed using the relays in that entry of the set. Furthermore, let $|m|$ denote the size of the rate matrix $\mathbf{L}_{m}$. A relay set and its corresponding solution, denoted as $\mathbf{t}_{m}$, is included as a potential solution if $\mathbf{t}_{m}$ satisfies the constraint, i.e.,

$$
\mathbf{t}_{m}>\mathbf{0}_{|m|}
$$

where $\mathbf{0}_{|m|}$ is the all-zero vector of size $|m|, \mathbf{0}_{|m|}=[0,0,0, \ldots 0]^{T}$ and the inequality operates on an element-by-element basis. Let the set $\mathcal{K}$ form the subset of $\mathcal{M}$ that comprises all potential solutions. Let $\mathbf{L}_{k}, \mathbf{t}_{k}$ and $|k|$ denote the rate matrix, its corresponding solution and size, respectively, for each entry of the set $\mathcal{K}$. Note that the number of active relays being considered in each entry is $|k|-1$. Finally, the 
optimum solution can be obtained by solving (16) for all possible combinations of active relays in the set $\mathcal{K}$ i.e.,

$$
\mathbf{t}^{*}=\max _{\mathcal{K}} \frac{\mathbf{L}_{k}^{-1} \mathbf{1}_{|k|}}{\mathbf{1}_{|k|}^{T} \mathbf{L}_{k}^{-1} \mathbf{1}_{|k|}}, \forall k=1, \ldots,|\mathcal{K}| .
$$

Given that entry $k^{*}$ corresponds to $\mathbf{t}^{*}$, the maximum achievable rate vector can thus be written as

$$
\mathbf{L}_{k^{*}} \mathbf{t}^{*}=\mathbf{L}_{k^{*}} \frac{\mathbf{L}_{k^{*}}^{-1} \mathbf{1}_{\left|k^{*}\right|}}{\mathbf{1}_{\left|k^{*}\right|}^{T} \mathbf{L}_{k^{*}}^{-1} \mathbf{1}_{\left|k^{*}\right|}}=\frac{\mathbf{1}_{\left|k^{*}\right|}}{\mathbf{1}_{\left|k^{*}\right|}^{T} \mathbf{L}_{k^{*}}^{-1} \mathbf{1}_{\left|k^{*}\right|}},
$$

and the maximum achievable rate, $R^{*}$, is

$$
R^{*}=\frac{1}{\mathbf{1}_{\left|k^{*}\right|}^{T} \mathbf{L}_{k^{*}}^{-1} \mathbf{1}_{\left|k^{*}\right|}},
$$

Note that the solution described above is equivalent to the iterative maximization in (6), and that removing a relay $r_{k}$ translates to removing the $k^{\text {th }}$ row and $(k+1)^{\text {th }}$ column from the rate matrix in (17). Removing the first relay, for example, reduces the rate matrix in (17) to

$$
\mathbf{L}_{N}=\left[\begin{array}{cccc}
L_{s r_{2}} & 0 & \ldots & 0 \\
L_{s r_{3}} & L_{r_{2} r_{3}} & \ldots & 0 \\
\vdots & \vdots & \ddots & 0 \\
L_{s d} & L_{r_{2} d} & \ldots & L_{r_{N} d}
\end{array}\right]
$$

Since $|\mathcal{M}|=2^{N}, 2^{N}$ possible solutions must be tested to find the global optimum.

\section{Numbering}

In Section III-A, we gave the solution to the optimization problem for a network with nodes numbered as in Figure 1. The numbering of the relay nodes impacts performance through causality: relay $r_{k}$ decodes information from relay $r_{k-1}$, but not vice-versa. A complete solution to the optimization problem must therefore take into account an optimal numbering scheme. In the worst case (in terms of computational power), an optimal solution can be obtained for a specific numbering scheme, and the truly optimal solution can be maximized over all possible numbering schemes.

Clearly, such an approach is impractical. Although a search for an optimal or effective sub-optimal solution is beyond the scope of this paper, we study the effects of numbering on the solution and resulting rate by considering some numbering schemes based on heuristics. We consider two approaches: numbering 
based on average channel conditions, and numbering based on instantaneous channel conditions.

1) Numbering based on average channel conditions: In the case of the linear network in Figure 1 , the numbering is trivial: node numbers increase away from the source and towards the destination. In the case of square network with nodes arranged in a grid, we consider two numberings which we refer to as Average Descending Numbering and Average Linear Numbering, shown in Figures 3 and 2 , respectively, for a $4 \times 4$ network.

- Average Descending numbering: node numbers increase towards the destination and downwards,

- Average Linear numbering: node numbers increase towards the destination but vertical numbering ensures that nodes closest to each other retain close numbering.

2) Numbering based on instantaneous channel conditions:

- Instantaneous $S-R_{k}$ numbering : node numbers increase with increasing source-relay channels. The first node has the best source-relay channel, the second node has the second-best source-relay channel, etc.

- Instantaneous $R_{k}-R_{m}$ numbering : nodes are numbered to maximize the channel between adjacent nodes. The first relay has the best source-relay channel. The second relay has the strongest $r_{1}$-relay channel. Numbers are assigned in this process to unoccupied relays. This heuristic is based on the notion that we should maximize the capacity of each $\left(R_{k}, R_{k+1}\right)$ hop.

- Random numbering : nodes are numbered randomly. This case evaluates the worst-case scenario and tests the robustness of the optimization to numbering.

These schemes are evaluated via simulations in Section $\mathrm{V}$, As we will see, the achievable rate is remarkably robust to the chosen numbering scheme.

\section{Partially Connected Network}

In this section we briefly discuss the more practical case of a partially connected network in which some links between the nodes in the network are unavailable. This is a generalization of the fully-connected network discussed in Section $\amalg$ II-A above. Such a network is more likely to represent a large scale network where, in any case, the solution in (19) would be computationally infeasible.

As an example, consider the two-relay network with the link between $r_{1}$ and $r_{2}$ is removed. The rate 
matrix thus becomes

$$
\mathbf{L}_{3}=\left[\begin{array}{ccc}
L_{s r_{1}} & 0 & 0 \\
L_{s r_{2}} & 0 & 0 \\
L_{s d} & L_{r_{1} d} & L_{r_{2} d}
\end{array}\right]
$$

Removing the link thus reduces the rank of this matrix by one, and the rate matrix is now non-invertible, eliminating the solution defined by $I_{1}=I_{2}=I_{3}$, where both relays are active. The optimal solution in this case is thus to select $r_{1}, r_{2}$, or not to relay. Note, however, that removing a link does not automatically lead to a non-invertible rate matrix. Consider, for example, the three-relay network with the link between $r_{1}$ and $r_{3}$ removed. The corresponding rate matrix

$$
\mathbf{L}_{4}=\left[\begin{array}{cccc}
L_{s r_{1}} & 0 & 0 & 0 \\
L_{s r_{2}} & L_{r_{1} r_{2}} & 0 & 0 \\
L_{s r_{3}} & 0 & L_{r_{2} r_{3}} & 0 \\
L_{s d} & L_{r_{1} d} & L_{r_{2} d} & L_{r_{3} d}
\end{array}\right]
$$

is full-rank and invertible.

The approach to the optimization problem for the case of the arbitrary connected network is that the same as for the fully-connected network, with the exception that the rate matrix $\mathbf{L}_{N+1}$ may not be invertible, in which case the corresponding solution is inadmissable. The remaining steps remain unchanged.

\section{IMPLEMENTATION WITH REDUCED COMPLEXITY}

The solution to the optimization problem in (4), (5) and (6) involves checking $2^{N}$ potential solutions. Although the process is conceptually simple, each solution involves the inverse of a rate matrix. In this section, we show how the optimization problem in the previous section can be significantly simplified using a recursive solution. This solution, which exploits the special structure of the rate matrix, greatly simplifies the matrix inversion, as well as reduces the number of possible solutions to check. Essentially, while the solution in Section III-A was a top-down approach, the approach we suggest here is bottom-up.

Consider a set of $p$ relays, $\mathcal{P}=\left\{r_{1}, r_{2}, \ldots, r_{p}\right\}, p \geq 0$, and its corresponding rate matrix $\mathbf{L}_{p+1}^{\mathcal{P}}$, solution vector $\mathbf{t}_{p+1}^{\mathcal{P}}$ and maximum rate (if available) $R^{\mathcal{P}}$. We note that if $p=0$ and the set is empty, the rate matrix and solution vector are constants, $L_{s d}$ and 1 , respectively. Denote as $\mathcal{P}^{\prime}$ the set $\mathcal{P}$ appended with another relay, i.e., $\mathcal{P}^{\prime}=\left\{r_{1}, r_{2}, \ldots, r_{p}, r_{p+1}\right\}$. Denote as $\mathbf{L}_{p+2}^{\mathcal{P}^{\prime}}, \mathbf{t}_{p+2}^{\mathcal{P}^{\prime}}$, and $R^{\mathcal{P}^{\prime}}$ the matrix, solution vector 
and rate corresponding to set $\mathcal{P}^{\prime}$.

Proposition 3: Given $\left(\mathbf{L}_{p+1}^{\mathcal{P}}\right)^{-1},\left(\mathbf{L}_{p+2}^{\mathcal{P}^{\prime}}\right)^{-1}$ can be obtained with computational complexity order of $O\left(p^{2}\right)$

Proof: For $p \geq 0$, the rate matrix $\mathbf{L}_{p+2}^{\mathcal{P}^{\prime}}$ can be written as

$$
\mathbf{L}_{p+2}^{\mathcal{P}^{\prime}}=\left[\begin{array}{c|c}
\mathbf{L}_{p+1}^{\mathcal{P}}(1: p, 1: p) & \mathbf{0}_{p \times 2} \\
\hline \mathbf{F}_{2 \times p} & \mathbf{T}_{2}
\end{array}\right],
$$

where $\mathbf{L}_{p+1}^{\mathcal{P}}(1: p, 1: p)$ denotes the first $p$ rows and columns of the rate matrix $\mathbf{L}_{p+1}^{\mathcal{P}}, \mathbf{0}_{p \times 2}$ is a $(p \times 2)$ matrix of zeros, $\mathbf{T}_{2}$ is a $(2 \times 2)$ lower- triangular matrix, and $\mathbf{F}_{2 \times p}$ is a $(2 \times p)$ fully-loaded matrix. Note that $\mathbf{L}_{p+1}^{\mathcal{P}}(1: p, 1: p)$ is triangular. Using the inverse of a partitioned matrix [39], $\left(\mathbf{L}_{p+2}^{\mathcal{P}^{\prime}}\right)^{-1}$ can be written as

$$
\left(\mathbf{L}_{p+2}^{\mathcal{P}^{\prime}}\right)^{-1}=\left[\begin{array}{c|c}
\left(\mathbf{L}_{p+1}^{\mathcal{P}}(1: p, 1: p)\right)^{-1} & \mathbf{0}_{p \times 2} \\
\hline-\mathbf{T}_{2}^{-1} \mathbf{F}_{2 \times p}\left(\mathbf{L}_{p+1}^{\mathcal{P}}(1: p, 1: p)\right)^{-1} & \mathbf{T}_{2}^{-1}
\end{array}\right]
$$

Note that $\left(\mathbf{L}_{p+1}^{\mathcal{P}}(1: p, 1: p)\right)^{-1}$ is the inverse of a partition of the triangular matrix $\mathbf{L}_{p+1}^{\mathcal{P}}$. Using the inverse of a partitioned matrix one more time, however, it is easy to see that

$$
\left(\mathbf{L}_{p+1}^{\mathcal{P}}(1: p, 1: p)\right)^{-1}=\left(\mathbf{L}_{p+1}^{\mathcal{P}}\right)^{-1}(1: p, 1: p)
$$

and thus

$$
\left(\mathbf{L}_{p+2}^{\mathcal{P}^{\prime}}\right)^{-1}=\left[\begin{array}{c|c}
\left(\mathbf{L}_{p+1}^{\mathcal{P}}\right)^{-1}(1: p, 1: p) & \mathbf{0}_{p \times 2} \\
\hline-\mathbf{T}_{2}^{-1} \mathbf{F}_{2 \times p}\left(\mathbf{L}_{p+1}^{\mathcal{P}}\right)^{-1}(1: p, 1: p) & \mathbf{T}_{2}^{-1}
\end{array}\right],
$$

and hence obtaining $\left(\mathbf{L}_{p+2}^{\mathcal{P}^{\prime}}\right)^{-1}$ is an $O\left(p^{2}\right)$ operation.

Using this above proposition, the solution vector $\mathbf{t}_{p+2}^{\mathcal{P}^{\prime}}$ of $\mathbf{L}_{p+2}^{\mathcal{P}^{\prime}}$ can be obtained from the solution vector $\mathbf{t}_{p+1}^{\mathcal{P}}$ of $\mathbf{L}_{p+1}^{\mathcal{P}}$ :

$$
\mathbf{t}_{p+2}^{\mathcal{P}^{\prime}}=\frac{\left(\mathbf{L}_{p+2}^{\mathcal{P}^{\prime}}\right)^{-1} \mathbf{1}_{p+2}}{\mathbf{1}_{p+2}^{T}\left(\mathbf{L}_{p+2}^{\mathcal{P}^{\prime}}\right)^{-1} \mathbf{1}_{p+2}}=\left[\begin{array}{c}
\mathbf{t}_{p+1}^{\mathcal{P}}(1: p) \\
\mathbf{t}_{p+2}^{\mathcal{P}^{\prime}}(p+1) \\
\mathbf{t}_{p+2}^{\mathcal{P}^{\prime}}(p+2)
\end{array}\right],
$$

where $\mathbf{t}_{p+1}^{\mathcal{P}}(1: p)$ represent the first $p$ entries of the already-calculated solution vector $\mathbf{t}_{p+1}^{\mathcal{P}}$, and $\mathbf{t}_{p+2}^{\mathcal{P}^{\prime}}(p+1)$ 
and $\mathbf{t}_{p+2}^{\mathcal{P}^{\prime}}(p+2)$ are the last two entries of the solution vector $\mathbf{t}_{p+2}^{\mathcal{P}^{\prime}}$ that remain to be calculated. $R^{\mathcal{P}^{\prime}}=$ $\frac{1}{\mathbf{1}_{p+2}^{T}\left(\mathbf{L}_{p+2}^{\mathcal{P}^{\prime}}\right)^{-1} \mathbf{1}_{p+2}}$ is the maximum achievable rate obtained using the set $\mathcal{P}^{\prime}$ of relays. The last two entries of the solution vector $\mathbf{t}_{p+2}^{\mathcal{P}^{\prime}}(p+1)$ and $\mathbf{t}_{p+2}^{\mathcal{P}^{\prime}}(p+2)$ can be written as

$$
\left[\begin{array}{c}
\mathbf{t}_{p+2}^{\mathcal{P}^{\prime}}(p+1) \\
\mathbf{t}_{p+2}^{\mathcal{P}^{\prime}}(p+2)
\end{array}\right]=R^{\mathcal{P}^{\prime}}\left[-\mathbf{T}_{2}^{-1} \mathbf{F}_{2 \times p}\left(\mathbf{L}_{p+1}^{\mathcal{P}}\right)^{-1}(1: p, 1: p) \mid \mathbf{T}_{2}^{-1}\right] \mathbf{1}_{(p+2) \times 1},
$$

With a corresponding achievable rate $R^{\mathcal{P}^{\prime}}$

$$
\begin{aligned}
R^{\mathcal{P}^{\prime}} & =\frac{1}{\mathbf{1}_{p+2}^{T}\left(\mathbf{L}_{p+2}^{\mathcal{P}^{\prime}}\right)^{-1} \mathbf{1}_{p+2}}=\left(\sum_{i j}\left(\mathbf{L}_{p+2}^{\mathcal{P}^{\prime}}\right)^{-1}(i, j)\right)^{-1}, \\
& =\left(\sum_{i, j}\left(\mathbf{L}_{p+1}^{\mathcal{P}}\right)^{-1}(i, j)-\sum_{i, j} \mathbf{T}_{2}^{-1} \mathbf{F}_{2 \times p}\left(\mathbf{L}_{p+1}^{\mathcal{P}}\right)^{-1}(1: p, 1: p)(i, j)+\sum_{i j} \mathbf{T}_{2}^{-1}(i, j)\right)^{-1},
\end{aligned}
$$

where we use $\sum_{i, j} \mathbf{A}(i, j)$ to denote the summation over all the elements of matrix $\mathbf{A}$.

Using the above, the optimization problem for a network of $N$ potential relays can be solved recursively as follows:

1) Determine the set of all potential relay combinations. Sequence the set as:

$$
\begin{array}{r}
\mathcal{M}=\left\{\left(r_{1}\right),\left(r_{1}, r_{2}\right),\left(r_{1}, r_{2}, r_{3}\right), \ldots\left(r_{1}, r_{2}, \ldots, r_{N}\right),\right. \\
\left(r_{1}, r_{3}\right),\left(r_{1}, r_{3}, r_{4}\right), \ldots,\left(r_{1}, r_{3}, \ldots, r_{N}\right), \\
\ldots \ldots \\
\left(r_{1}, r_{N}\right), \\
\left(r_{2}\right),\left(r_{2}, r_{3}\right),\left(r_{2}, r_{3}, r_{4}\right), \ldots\left(r_{2}, r_{3}, \ldots, r_{N}\right), \\
\left(r_{2}, r_{4}\right),\left(r_{2}, r_{4}, r_{5}\right), \ldots,\left(r_{2}, r_{4}, \ldots, r_{N}\right), \\
\ldots \ldots \\
\left(r_{2}, r_{N}\right), \\
\ldots \\
\left.\left(r_{N-1}, r_{N}\right)\right\} .
\end{array}
$$


Note that each "row" of $\mathcal{M}$ is a subset of relay combinations in which each element is formed from the previous element by adding a relay.

2) In each "row", obtain the rate matrix, its respective optimized time allocation vector and achievable rate for each element (i.e., relay combination) recursively using (28), (29), (30) and (31).

3) Check that for each particular set $\mathcal{P}$ of $p$ relays, the solution $\mathbf{t}_{p}$ and achievable rate $R_{p}$ satisfies the constraints:

$$
\begin{gathered}
R^{\mathcal{P}} \geq 0, \\
\mathbf{t}_{p+1}^{\mathcal{P}}>\mathbf{0}_{p+1} .
\end{gathered}
$$

- If both constraints are satisfied, place the solution in the potential set of valid solutions $\mathcal{K}$, advance elements and return to step (1).

- If (33) is not satisfied, check which element of the the allocation vector $\mathbf{t}_{p}$ does not satisfy the constraint.

- If any of the first $(p-1)$ entries of $\mathbf{t}_{p}$ are less than zero, i.e., $\mathbf{t}_{p}\left(1: p-1<\mathbf{0}_{p-1}\right)$, this constraint will not be satisfied for any other relay combinations in this "row". Advance rows and return to item (1).

- If the constraint is not satisfied by either of the last two items in the solution vector, discard the solution but check the other elements in the "row".

4) From the set $\mathcal{K}$, pick the highest achievable rate and its corresponding time allocation.

The recursive algorithm given above simplifies the optimization problem in two ways:

1) It reduces the computation load of determining successive matrix inverses by writing each matrix inverse as a function of another, already known, matrix inverse, and two other matrices obtained through simple matrix multiplication.

2) It may eliminate infeasible solutions by discarding relay combinations which do not satisfy constraints. For example, if the relay combination $\left(r_{1}, r_{2}, r_{3}\right)$ does not satisfy the constraints, the combination $\left(r_{1}, r_{2}, r_{3}, r_{4}\right)$ may be automatically discarded.

\section{A. Complexity and Number of Operations}

In the next paper we will compare relay selection schemes partly on computational complexity. In this section we calculate this complexity, which also quantifies the computational savings of the recursive 
scheme presented above in Section IV]

The complexity of the recursive scheme is bounded by complexity of matrix multiplication. The number of operations (multiplications and additions) required in the product of two matrices of size $(m, n)$ and $(n, p)$ is $2 m p n-m p$ [40], and the number of operations required for the product of a matrix of size $(m, n)$ with a square, size- $n$ diagonal matrix is

$$
m\left(\sum_{k=0}^{n-1} k+\sum_{k=1}^{n} k\right)=m n^{2} .
$$

We now calculate the number of operations required for each rate matrix of size $(q+1)$, corresponding to the set $\mathcal{Q}^{\prime}$ of $q$ relays. The calculation of the matrix fundamental to the recursive algorithm,

$$
\left(\mathbf{L}_{q+1}^{\mathcal{Q}^{\prime}}\right)^{-1}=-\mathbf{T}_{2}^{-1} \mathbf{F}_{2 \times(q-1)}\left(\mathbf{L}_{q}^{\mathcal{Q}}\right)^{-1}(1: q-1,1: q-1)
$$

requires a total of $2 q^{2}+2 q+1$ operations, broken down as:

1) $-\mathbf{T}_{2}^{-1} \rightarrow 5$ operations,

2) $-\mathbf{T}_{2}^{-1} \mathbf{F}_{2 \times(q-1)}=\mathbf{A}_{2 \times(q-1)} \rightarrow 6(q-1)$ operations using $2 m p n-m p$,

3) $\mathbf{A}_{2 \times(q-1)}\left(\mathbf{L}_{q}^{\mathcal{Q}}\right)^{-1}(1: q-1,1: q-1) \rightarrow 2(q-1)^{2}$ operations, using (34).

From (31), the number of operations required to calculate $R^{\mathcal{Q}^{\prime}}$ is $q^{2}+2 q+4$. Using (30), the number of operations required to update the solution vector is $1+2(q+1)=2 q+3$. Summing the above, we obtain the total number of operations required in one iteration of the resource allocation algorithm:

$$
\mathrm{Op}(q)=\left(2 q^{2}+2 q+1\right)+\left(q^{2}+2 q+4\right)+(2 q+3)=3 q^{2}+6 q+8
$$

Note that the complexity order of calculating each rate and solution vector is $O\left(q^{2}\right)$. Without the recursion, this complexity is of order $O\left(q^{3}\right)$, resulting from the inverse of the rate matrix. The recursion thus introduces significant savings in terms of complexity.

We now calculate the worst-case total number of operations required by the resource allocation algorithm. In the worst case, the algorithm cycles through $2^{N}$ operations consisting of $\left(\begin{array}{l}N \\ q\end{array}\right)$ sets of $q$ relays which require $3 q^{2}+6 q+8$ operations. The total worst case number of operations is therefore

$$
\sum_{q=1}^{N}\left(\begin{array}{l}
N \\
q
\end{array}\right)\left(3 q^{2}+6 q+8\right) .
$$

This calculation could be rendered more precise if it were possible to account for the savings obtained in 
Section IV which eliminates some infeasible solutions a priori by discarding relay combinations known to not satisfy the constraints. The probability of this occurring for particular channel realizations is unfortunately very difficult to compute, and we thus show only the worst-case result.

\section{Simulations}

In this section, we present results of the resource allocation scheme discussed in Section with 1 to 6 relays arranged linearly, and 4 and 9 nodes arranged in a grid. The figure of merit is the achievable rate $R_{a}$ with an outage probability of $10^{-3}$, i.e. $\operatorname{Pr}\left[R^{*}<R_{a}\right]=10^{-3}$. A closed form expression for the outage probability of optimized cooperation is very complicated and beyond the scope of the paper. The outage probability and rate are thus obtained numerically.

The relays are equispaced on a line between the source and destination, as in Figure 1, and we use an attenuation exponent of $p_{a}=2.5$. This choice is motivated by the application of static mesh-nodes installed on posts; transmissions between such nodes should undergo little shadowing and a lower attenuation exponent. From 60000 fading realizations we obtain the cumulative density function of the instantaneous rate $F_{R}(r)$. The outage rate is the rate for which the probability of outage is $10^{-3}$, i.e., $F_{R}^{-1}\left(10^{-3}\right)$.

Figure 4 and 5 plot the outage rate as a function of the average end-to-end SNR, $\frac{P}{N_{0} W}$, for optimized and non-optimized cooperation, respectively. The rate for the optimized cooperation is obtained from (19). Non-optimized cooperation uses equal time allocation, i.e., the rate for a particular relay set is simply the minimum of the mutual information at each node. Non-optimized cooperation, however, does optimally select relays by choosing the best, in terms of rate, of the $2^{N}$ relay combinations. Comparing Figure 4 and Figure 5 shows that optimizing resources increases rates significantly, as expected. The outage rate increases as a function of nodes available to relay. We also note the typical phenomenon of decreasing marginal returns: the gains of adding each additional relay decreases with increasing number of relays.

Figures 6 and 7 show the average number of relays that are active from the set of potential relays for optimized and non-optimized cooperation. For each network size, this number is a decreasing function. Interestingly, the number of active relays decreases much faster for non-optimized as compared to optimized cooperation, suggesting that optimizing resources distributes the relaying burden more effectively.

To test the effect of geometry on the outage rate, we compare the rates obtained by optimizing resources and the placing relays on a line, as in Figure 4 to those obtained by placing the relays on a regular square grid. We number the relays in the grid in ascending order downwards and towards the source; a derivation of the optimal numbering is beyond the scope of this paper. The results are demonstrated in Figure 8 
where we place 4 and 9 relays on a $2 \times 2$ and $3 \times 3$ square grid. As shown in the figure, the rate for the linear constellation is significantly higher than that obtained by the grid constellation, suggesting that the path-loss incurred by traversing all the nodes laterally results in non-negligible performance loss.

We evaluate the performance of the numbering schemes discussed in Section $\amalg$ II-C in Figure 9 . The four schemes, including two based on average channel conditions and two based on instantaneous channel conditions, exhibit indistinguishable performance in terms of rate. There is an expected drop in rate with random numbering, though we note that this drop is no more than approximately 0.25 bits/channel use. The algorithm is thus quite robust to numbering schemes.

Figure 9 also shows the outage rate for a network with randomly placed nodes. Here the node locations are chosen from a uniform distribution over an area equivalent to that of the square grid. The internode channels are obtained as before. This example eliminates possible dependencies of the results obtained earlier on the chosen array geometry. The numbering here is based on the instantaneous $S-R_{k}$ channels. In such a random network, as expected, the available outage rate is lower than in a square grid network; however, at higher SNR levels this difference disappears. Again, the significant gains due to resource allocation are clear.

In Figure 9 we also compare the effect of numbering when used without resource allocation, and show only the case of instantaneous $S-R_{k}$ numbering and random numbering. The improvement from instantaneous over random numbering in this case is less than $0.1 \mathrm{bits} / \mathrm{channel}$ use. The robustness of the numbering scheme thus increases by eliminating time optimization. To gain insight into this phenomenon, in Figure 10 we plot the average number of active users for the instantaneous and random numbering schemes with and without resource allocation. We first observe that the instantaneous numbering scheme uses more relays than the random numbering scheme when resource allocation is used, and that this difference is constant over the SNR region of interest. Without resource allocation, on the other hand, the number of relays used when using instantaneous and random numbering decreases quickly and is constant for SNR values higher than $10 \mathrm{~dB}$. It is clear from this figure that the difference in rate performance between instantaneous and random numbering is an increasing function of the number of selected relays. Because so few relays are selected without resource allocation, the effect of the numbering scheme is negligible. The influence of the numbering scheme increases when time allocation is introduced, increasing the number of relays used for both numbering schemes and increasing the sensitivity to the numbering scheme. This sensitivity increases slowly, however, and is negligible for the various numbering schemes 
based on heuristics.

\section{CONCLUSIONS}

In this paper, we determined the optimal channel resource allocation, in terms of time allocation, for the $N$-node cooperative diversity, multihop network using DF and independent codebooks. For a particular network, i.e., set of potential relays, the unique solution for a particular relay numbering scheme is obtained by taking the inverse of the triangular rate matrix, and the optimal solution is found by maximizing over the rate for each possible network, given its maximum size. Through simulations, however, the optimization is shown to be robust to the numbering scheme. We show that by exploiting the special structure of the rate matrix, the optimization can be performed in a recursive fashion which decreases the computation load of the rate matrix inverse and the number of required iterations. Node selection is inherent to the optimization strategy. Simulation results show significant gains in achievable rate due to resource allocation, but diminishing marginal returns as a function of network size. Furthermore, we show a significant benefit to arranging the nodes in a linear, as opposed to a grid, constellation.

\section{REFERENCES}

[1] A. Sendonaris, E. Erkip, and B. Aazhang, "User cooperation diversity - part I, II," IEEE Trans. Commun., vol. 51, pp. 1927 - 1948, November 2003.

[2] J. N. Laneman and G. W. Wornell, "Distributed space-time-coded protocols for exploiting cooperative diversity in wireless networks," IEEE Trans. Inf. Theory, vol. 49, pp. 2415 - 2425, October 2003.

[3] E. Beres and R. Adve, "Selection cooperation in multi-source cooperative networks," IEEE Trans. Wireless Communications, vol. 7, pp. 118 - 127, Januaryr 2008.

[4] Y. Yao, X. Cai, and G. B. Giannakis, "On energy efficiency and optimum resource allocation of relay transmissions in the low-power regime," IEEE Trans. Wireless Commun., vol. 4, pp. 2917- 2927, November 2005.

[5] E. G. Larsson and Y. Cao, "Collaborative transmit diversity with adaptive radio resource and power allocation," IEEE Commun. Lett., vol. 9, pp. 511-513, June 2005.

[6] D. Gunduz and E. Erkip, "Opportunistic cooperation by dynamic resource allocation," IEEE Trans. Wireless Commun., vol. 6, pp. 1446 - 1454, April 2007.

[7] J. Yang, D. Gunduz, D. Brown III, and E. Erkip, "Resource allocation for cooperative relaying," in Proc. of Conference on Information Sciences and Systems (CISS 2008), March 2008.

[8] A. Host-Madsen and J. Zhang, "Capacity bounds and power allocation for wireless relay channels," IEEE Trans. Inf. Theory, vol. 51, pp. 2020 - 2040, June 2005.

[9] L. Xie and X. Zhang, "Opportunistic cooperation for quality of service provisionings over wireless relay networks," in Proc. of IEEE Int. Conf. on Communications, June 2007.

[10] Y. Ning, T. Hui, C. Shasha, and Z. Ping, "An adaptive frame resource allocation strategy for TDMA-based cooperative transmission," IEEE Commun. Lett., vol. 11, pp. 417 - 419, May 2007. 
[11] M. Ibrahimi and B. Liang, "Efficient power allocation in cooperative OFDM system with channel variation," in IEEE International Conference on Communications (ICC), May 2008.

[12] P. A. Anghel, M. Kaveh, and Z. Q. Luo, "Optimal relayed power allocation in interference-free non-regenerative cooperative systems," in Proc. of IEEE Workshop on Signal Processing Advances in Wireless Communications, July 2004.

[13] _ _ "An efficient algorithm for optimum power allocation in a decode-and-forward cooperative system with orthogonal transmissions," in Proc. of the IEEE International Conference on Acoustics, Speech and Signal Processing ICASSP, May 2006.

[14] Y. Liang, V. Veeravalli, and H. V. Poor, "Resource allocation for wireless fading relay channels: Max-min solution," IEEE Trans. Inf. Theory, vol. 53, pp. 3432 - 3453, October 2007.

[15] J. Boyer, D. Falconer, and H. Yanikomeroglu, "Multihop diversity in wireless relaying channels," IEEE Trans. Commun., vol. 52, pp. 1820 - 1830, October 2004.

[16] A. K. Sadek, W. Su, and K. J. R. Liu, "Multinode cooperative communications in wireless networks," IEEE Trans. Signal Process., vol. 55, no. 1, pp. 341-355, January 2007.

[17] Z. Yang and A. Host-Madsen, "Routing and power allocation in asynchronous Gaussian multiple-relay channels," EURASIP Journal on Wireless Communications and Networking, 2006.

[18] J. Zhang and T. M. Lok, "Performance comparison of conventional and cooperative multihop transmission," in Proc. of the Wireless Communications and Networking Conference (WCNC), April 2008.

[19] S.-H. Chen, U. Mitra, and B. Krishnamachari, "Cooperative communication and routing over fading channels in wireless sensor network," in Proc. of the IEEE International Conference on Wireless Networks,Communications, and Mobile Computing (WirelessCom), June 2005 .

[20] X. Fang, T. Hui, Z. Ping, and Y. Ning, “Cooperative routing strategies in Ad Hoc networks," in Proc. of the IEEE Vehicular Technology Conference (VTC-Spring 2005), June 2005.

[21] A. Khandani, J. Abounadi, E. Modiano, and L. Zhang, "Cooperative routing in wireless networks," in Proc. of Allerton Conference on Communications, Control and Computing, October 2006.

[22] F. Li, A. Lippman, and K. Wu, "Minimum energy cooperative path routing in wireless networks: An integer programming formulation," in Proc. of the 63th IEEE Vehicular Technology Conference (IEEE VTC '2006), May 2006.

[23] Y. Yuan, Z. He, and M. Chen, "Virtual MIMO-based cross-layer design for wireless sensor networks," IEEE Transactions on Vehicular Technology, vol. 55, pp. 856- 864, May 2006.

[24] Y. Yuan, M. Chen, and T. Kwon, "A novel cluster-based cooperative MIMO scheme for multi-hop wireless sensor networks," EURASIP Journal on Wireless Communications and Networking, vol. 2006, pp. Article ID 72 493, 9 pages, 2006.

[25] V. Srinivasan, P. Nuggehalli, C.-F. Chiasserini, and R. Rao, "An analytical approach to the study of cooperation in wireless Ad Hoc networks," IEEE Trans. Wireless Commun., vol. 4, no. 2, pp. 722 - 733, March 2005.

[26] L. Ong and M. Motani, "Optimal routing for decode-and-forward based cooperation in wireless networks," in Proc. of the IEEE Conference on Sensor, Mesh and Ad Hoc Communications and Networks (SECON), June 2007.

[27] A. Aksu and O. Ercetin, "Reliable multi-hop routing with cooperative transmissions in energy-constrained networks," IEEE Trans. Commun., vol. 7, no. 8, pp. 2861-2865, August 2008.

[28] Y. Zhao, R. S. Adve, and T. J. Lim, "Improving amplify-and-forward relay networks: Optimal power allocation versus selection," IEEE Trans. Wireless Commun., vol. 6, pp. 3114 - 3123, August 2007.

[29] A. Bletsas, A. Khisti, D. Reed, and A. Lippman, "A simple cooperative diversity method based on network path selection," IEEE J. Sel. Areas Commun., vol. 24, pp. 659-672, March 2006. 
[30] A. Bletsas, H. Shin, and M. Z. Win, “Cooperative communications with outage-optimal opportunistic relaying," IEEE Trans. Wireless Commun., vol. 6, no. 9, pp. 3450-3460, September 2007.

[31] A. Chakrabarti, E. Erkip, A. Sabharwal, and B. Aazhang, "Code designs for cooperative communication," IEEE Signal Processing Magazine, vol. 24, pp. 16 - 26, September 2007.

[32] G. Kramer, M. Gastpar, and P. Gupta, "Cooperative strategies and capacity theorems for relay networks," IEEE Trans. Inf. Theory, vol. 51, no. 9, pp. 3037-3063, September 2005.

[33] L. L. Xie and P. R. Kumar, “An achievable rate for the multiple-level relay channel,” IEEE Trans. Inf. Theory, vol. 51, no. 4, pp. 1348 $-1358,2005$

[34] P. Gupta and P. R. Kumar, "Towards an information theory of large networks: an achievable rate region," IEEE Trans. Inf. Theory, vol. 49, no. 8, pp. 1877-1894, August 2003.

[35] S. Avestimehr, S. N. Diggavi, and D. N. C. Tse, "Approximate characterization of capacity in Gaussian relay networks," in Wireless Communications and Mobile Computing Conference (IWCMC), August 2008.

[36] A. Reznik, S. R. Kulkarni, and S. Verdu, "Degraded Gaussian multirelay channel: capacity and optimal power allocation," IEEE Trans. Inf. Theory, vol. 50, no. 12, pp. 3037-3046, December 2004.

[37] P. Razaghi and W. Yu, "Parity forwarding for multiple-relay networks," IEEE Trans. Inf. Theory, 2007, submitted. Available online at http://www.comm.utoronto.ca/ weiyu/publications.html

[38] P. Rost and G. Fettweis, "Achievable rates in Gaussian half-duplex multiple relay networks," in Proceedings if IEEE Global Conference on Communications (Globecom), December 2008.

[39] R. A. Horn and C. R. Johnson, Matrix Analysis. Cambridge University Press, 1985.

[40] I. Kaporin, “A practical algorithm for faster matrix multiplication,” Numerical Linear Algebra with Applications, vol. 6, pp. 687 - 700, 1999. 


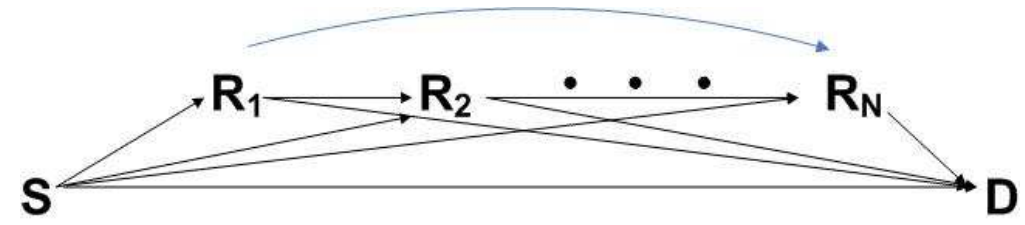

Fig. 1. Location of the relays with respect to the source and destination.

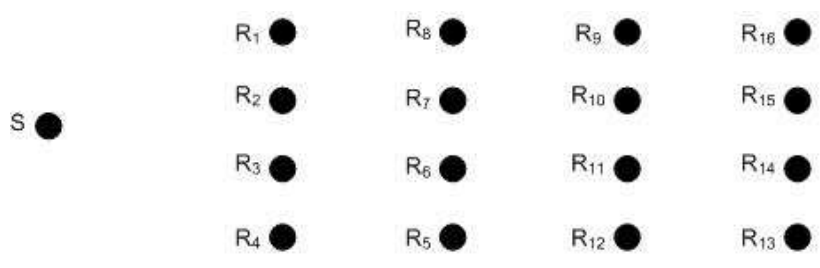

D

Fig. 2. Numbering in a square $4 \times 4$ network in linear order

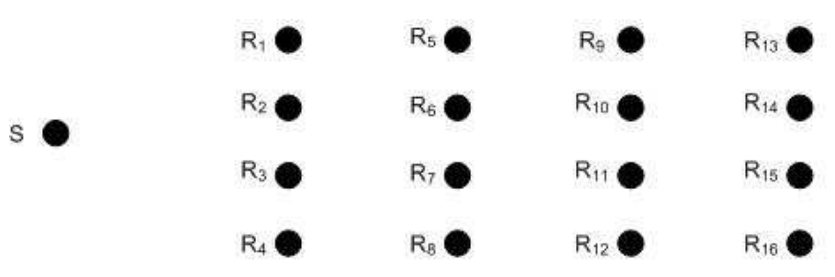

Fig. 3. Numbering in a square $4 \times 4$ network in descending order

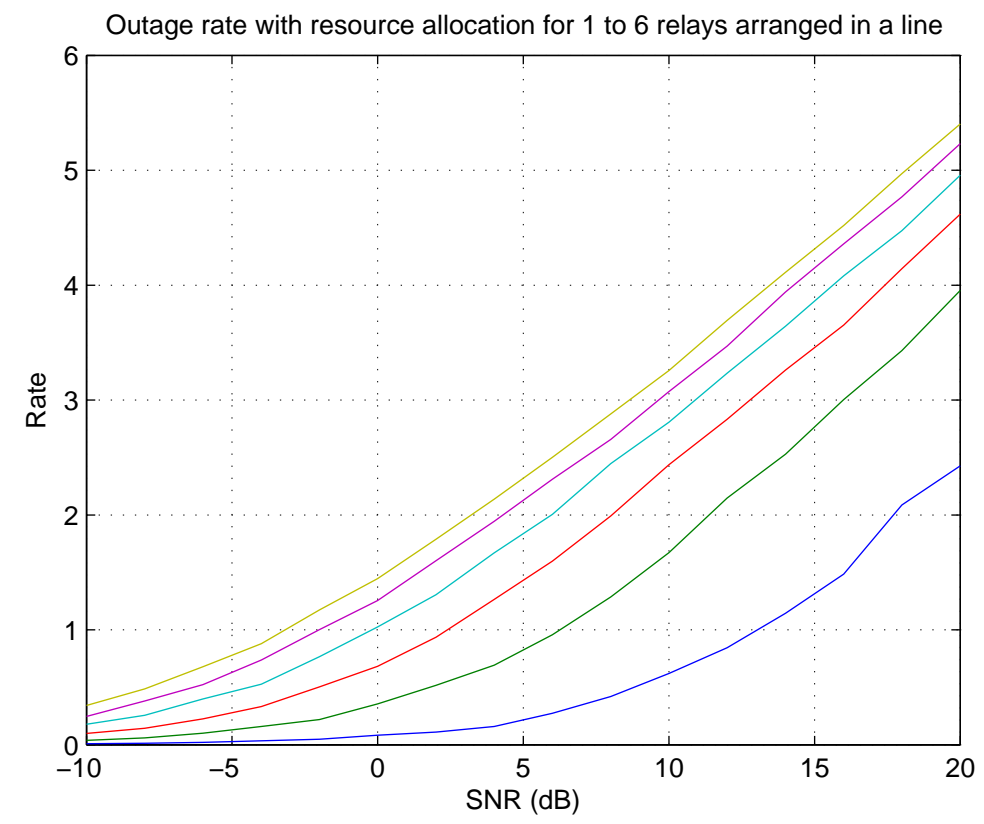

Fig. 4. Outage rate vs. SNR using $1, \ldots 6$ potential relays and with resource allocation. 


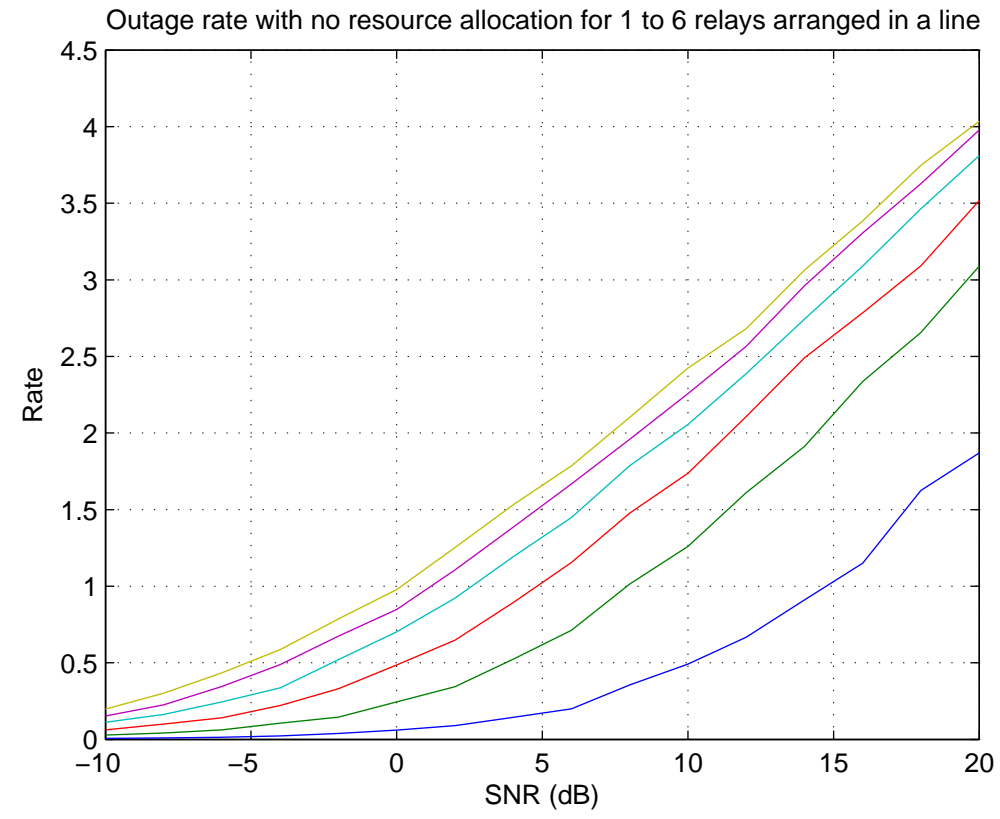

Fig. 5. Outage rate vs. SNR using $1, \ldots 6$ potential relays and without resource allocation. 


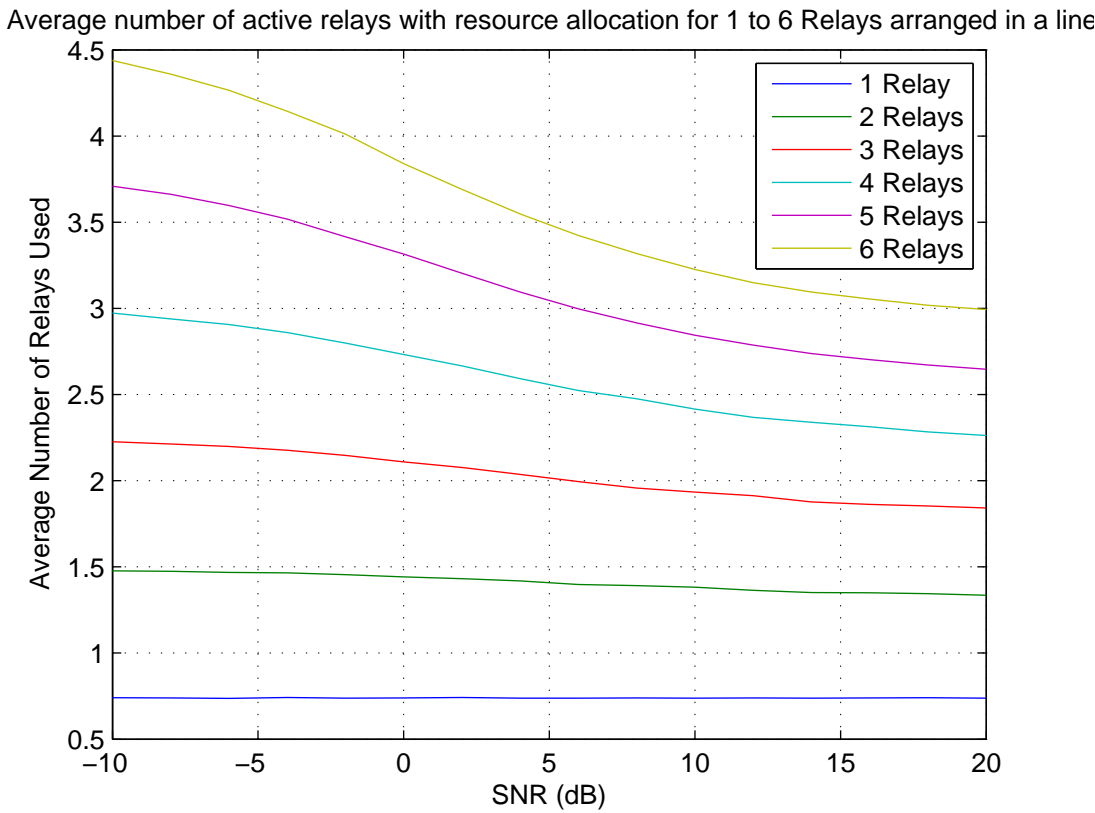

Fig. 6. Average number of active relays with $1, \ldots 6$ potential relays and with resource allocation.

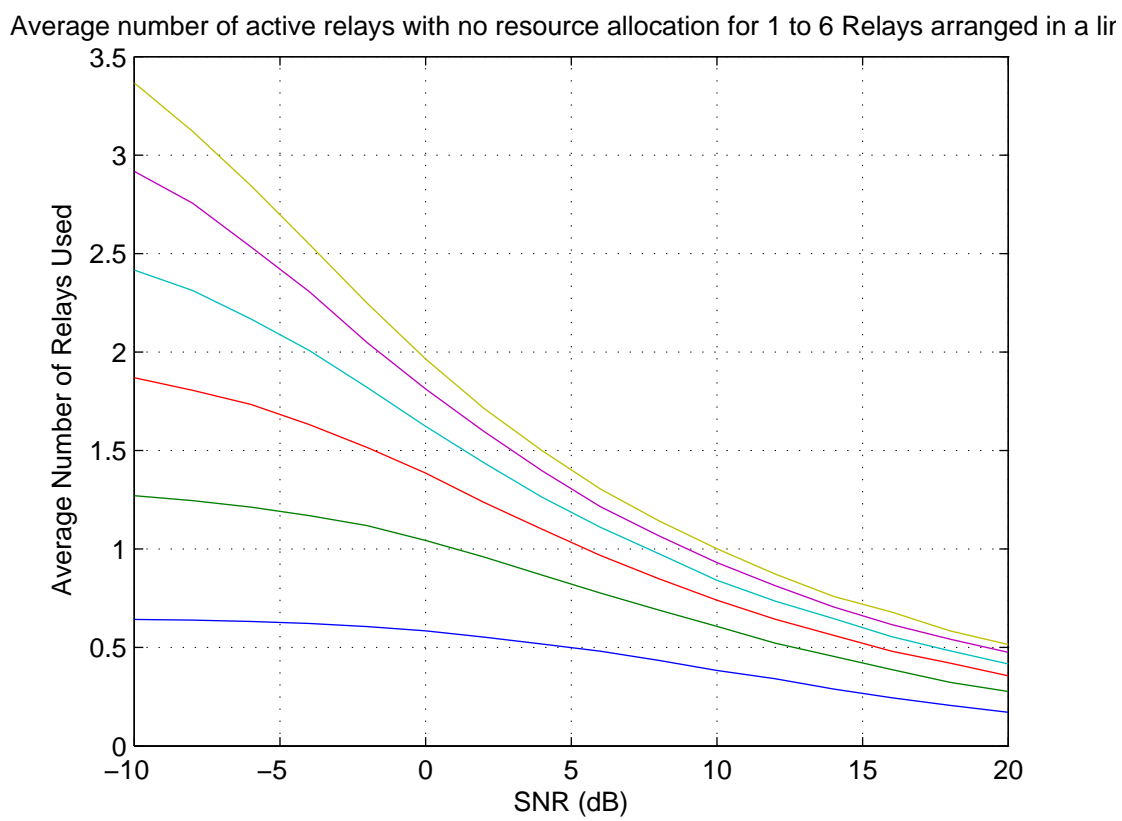

Fig. 7. Average number of active relays with $1, \ldots 6$ potential relays and without resource allocation. 


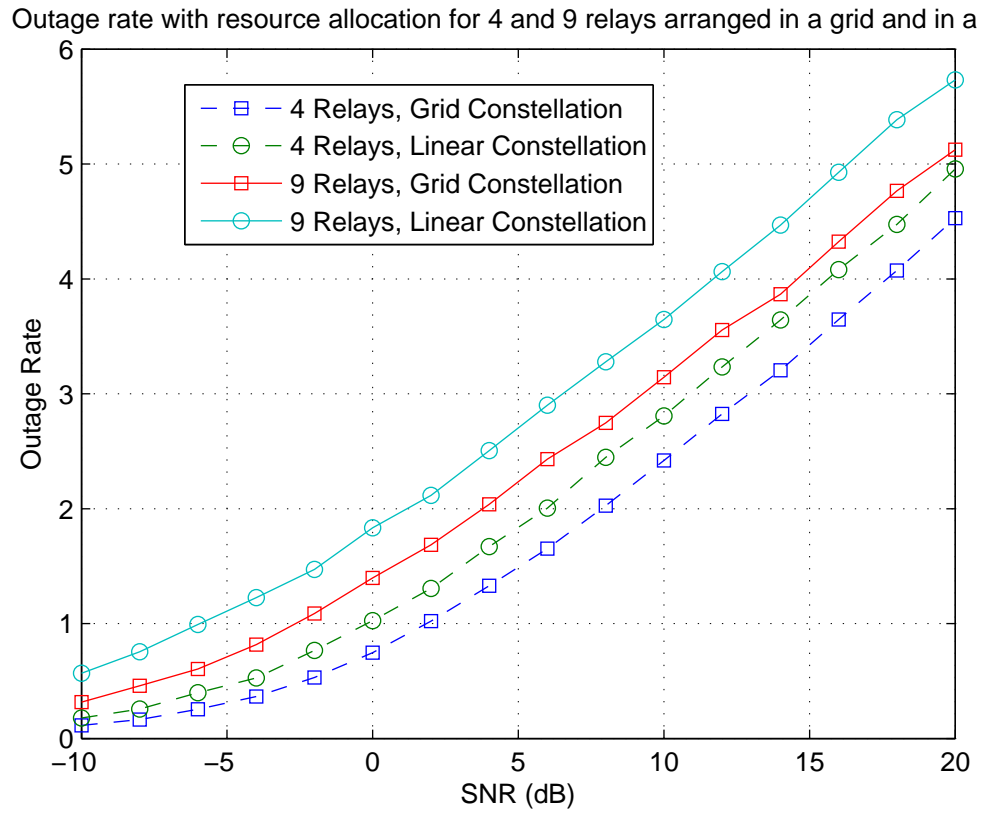

Fig. 8. Outage rate vs. SNR using resource allocation and for 4 and 9 relays arranged in a grid and in a line.

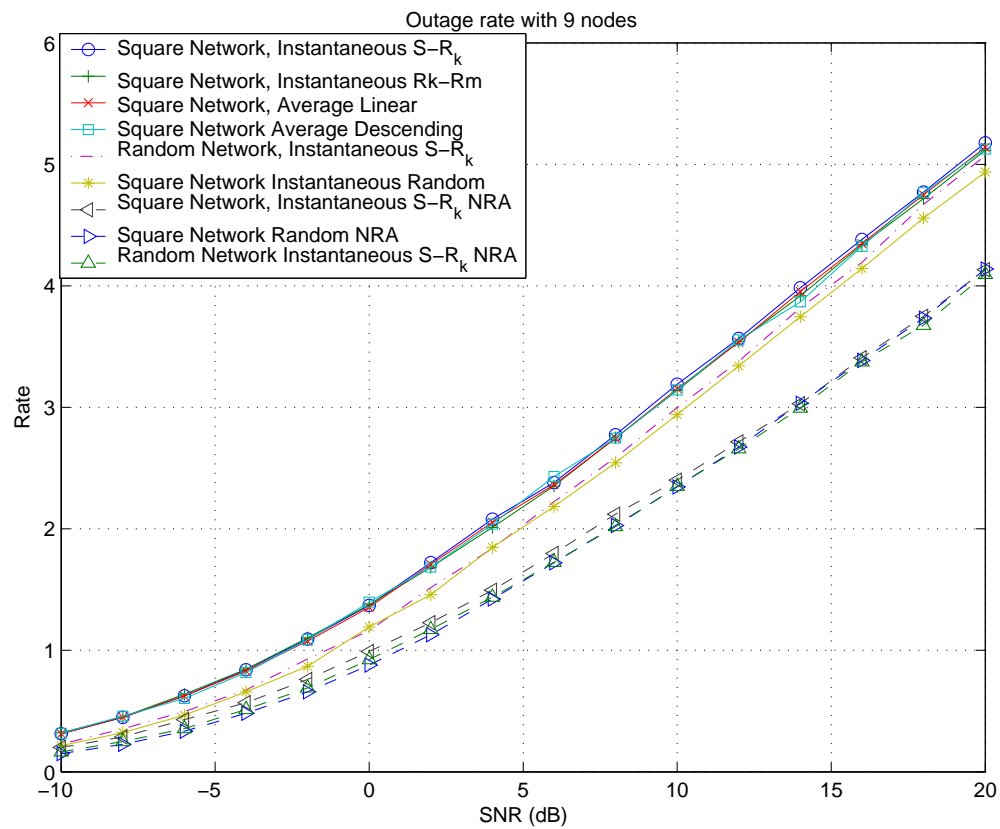

Fig. 9. Outage rate vs. SNR using resource allocation and for various numbering schemes for 9 potential nodes arranged in a grid 


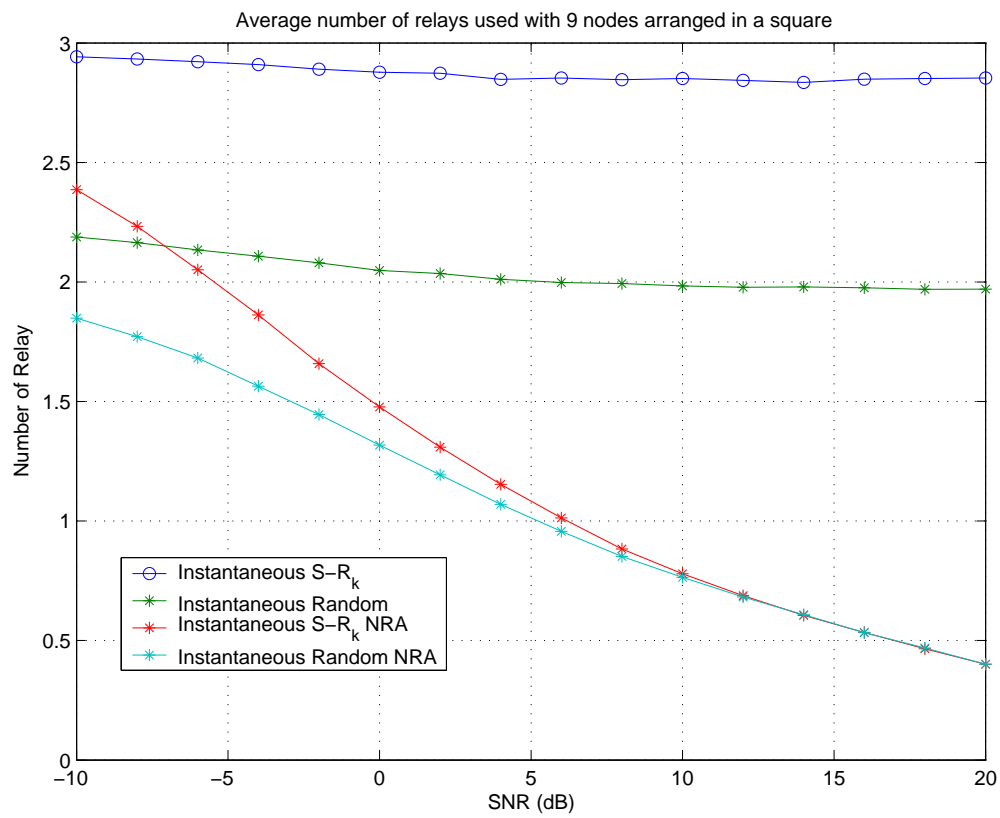

Fig. 10. Average number of active relays using resource allocation and with various numbering schemes for 9 potential nodes arranged in a grid 